Chapter 2:

Research Papers 



\title{
Two Methods for Improving Inductive Logic Programming Systems
}

\author{
Irene Stahl $^{1}$, Birgit Tausend ${ }^{1}$ and Rüdiger Wirth ${ }^{2}$ \\ 1 Fakultät Informatik, Universitāt Stuttgart, Breitwiesenstr. 20-22, \\ D-7000 Stuttgart 80 \\ 2 Research Institute for Applied Knowledge Processing, P. O. Box 2060, D-7900 Ulm
}

\begin{abstract}
In this paper we describe two methods for improving systems that induce disjunctive Horn clause definitions. The first method is the well-known use of argument types during induction. Our novel contribution is an algorithm for extracting type information from the example set mechanically.

The second method provides a set of clause heads partitioning the example set in disjuncts according to structural properties. Those heads can be used in top-down inductive inference systems as starting point of the general-to-specific search and reduce the resulting space of clause bodies.
\end{abstract}

\section{Introduction}

Inductive inference of Horn clause definitions from ground facts faces the problem of a very large, in general infinite hypothesis space. No matter whether it is searched from general to specific (top-down) or from specific to general (bottomup) there are problems with inefficiency or even intractability.

One simple way to reduce the space of possible clauses is the use of argument types for predicates. Clauses that contain a variable occurring at argument positions with conflicting types need not be considered. Due to its simple and efficient realizability typing has been employed from the very beginning of inductive inference in first order logic, e.g. in Shapiro's Model Inferece System MIS [Sha83]. However, all systems using argument types require user-supplied type declarations.

We show that the argument types of a predicate can be determined automatically from its example set. The problem is an instance of the general inductive inference problem and can be solved deterministically.

Apart from reducing the space of possible clauses, argument types can be used to discover structural commonalities among the examples. We propose a method for determining a set of clause heads that partitions the example set according to structural properties.

For top-down approaches to inductive inference, the knowledge about those heads helps in inducing disjunctive definitions, i.e. definitions consisting of more than one clause. Systems performing a general-to-specific search like MIS [Sha83] and Foll [Qui90] start their search for each single clause with the most general 
form of the target predicate as clause head and then successively add body literals or instantiate variables with complex terms. In doing so they look both for discriminating and characterizing information at the same time. The size of the search space can be reduced by seperating these two tasks into subtasks.

Most of the discriminating information is structural, e.g. the difference between base case and recursive case of a predicate. The method we propose first learns discriminating information by partitioning the example set according to structural properties. These structural properties are expressed as predicates which form the heads of clauses covering a disjunct. In the second step we induce clauses characterizing each disjunct by specialising the clause heads derived in step one. This technique provides two advantages: the search is started with less general heads and the structure of the terms in the head yield powerful constraints for the search, which for instance can be employed during predicate invention [WO91].

Both methods have been incorporated in an experimental implementation called INDICo (INduction of Dlsjunctive COncepts). This implementation has been used to measure the improved efficiency against MIs [Sha83], FoIL [Qui90] and CHAM [Kij92].

This paper is organized as follows. First, we discuss the algorithm for determining the type restrictions. Secondly, the method for computing clause heads for the target predicate is described. In the following section we give an overview of our experimental system INDICO. Finally, we present some experimental results obtained with INDICO and conclude.

\section{Determining the Argument Types}

Determining argument types can be viewed as inductive inference of Horn clause definitions from facts. Given the sets $E$ and $N$ of ground facts, the positive and negative examples, a logic program $B$ as background knowledge and mode declarations [Sha83] for each predicate in $E, N$ and $B$, the task of inductive inference is to find a logic program $P$ such that $B \cup P \vdash_{S L D} E$ and $B \cup P \forall S L D N$. $P$ is an extension of the given theory $B$ such that all examples in $E$, but none in $N$, are covered by the extended theory. As we restrict our algorithms to positive examples, we consider only the set $E$. Furthermore, we assume that $E$ contains only examples for one target predicate $p / n$. However, the generalisation of our algorithms to the case that $E$ contains examples of different predicates is straightforward.

For describing argument types of a predicate $p / n$ we use the following notation:

$$
\text { type_restriction }\left(p\left(X_{1}, \ldots, X_{n}\right),\left\{\text { type }_{1}\left(X_{1}\right), \ldots, \text { type }_{n}\left(X_{n}\right)\right\}\right)
$$

where each type $_{i}\left(X_{i}\right)$ is defined by a set of Horn clauses, each satisfying the following syntactic rules:

- the head literal is of the form type $i(T)$, where $T$ is a term. 
- every body literal is of the form $p(X)$, where $X$ is a variable and $p$ is either a type type $e_{l}$ or a predicate in the background knowledge $B_{\text {type }}$. We restricted $B_{\text {type }}$ to the standard definitions of atom, number and atomic.

- every variable occurs exactly once in the head and once in the body of the clause.

Example 1. If the example set is $\{$ append $(\square, \square, \square)$, append $([1, a, 2],[3],[1, a, 2,3])$, append $([p],[,[p]) \ldots .$.$\} , then the corresponding type restriction is$

$$
\begin{aligned}
& \text { type_restriction(append } \left.(X, Y, Z),\left\{t_{1}(X), t_{1}(Y), t_{1}(Z)\right\}\right) . \\
& t_{1}(\emptyset) \text {. } \\
& t_{1}([X \mid Y]) \leftarrow \text { atomic }(X), t_{1}(Y) .
\end{aligned}
$$

The type $t_{1}$ defines recursive lists of atomic terms with the empty list [ as base case.

The rules restrict type definitions within our framework to regular unary logic (RUL) programs [YS91]. As the extensions of predicates defined by RUL-programs are regular sets, inclusion and equivalence of different types can easily be checked. This is an important precondition for the use of argument types during induction.

$B_{\text {type }}$ need not be restricted to atom, number and atomic, but may be extended by further unary predicates expressing semantic restrictions as e.g. odd_number. However, there must be an effective method that, given a set of terms, returns the unique most specific predicate $p$ within $B_{\text {type }}$ that is valid for all terms in the set, and fails if none exists. Therefore, the extensions of the predicates in $B_{\text {type }}$ have to constitute a complete lattice with respect to set union and intersection. Allowing arbitrary unary or $n$-ary $(n>1)$ predicates in $B_{\text {type }}$ leads to non-regular types. For those, inclusion and equivalence may be undecidable.

For each argument position $i$ of the target predicate, the starting point of our algorithm is the set of $i$-th arguments of the examples. Let $A$ be this set for a position $i$ and set

$$
E_{A}=\left\{\operatorname{type}_{A}(T) \mid T \in A\right\} .
$$

Then the task of the algorithm for determining the argument types is to find a set of clauses $P_{t y p e_{A}}$ such that

$$
B_{\text {type }} \cup P_{\text {typeA }} \vdash E_{A} \text {. }
$$

Thus determining argument types is a special case of inducing Horn clause definitions from positive examples. Both the background knowledge $B_{\text {type }}$ and the form of the target clauses $P_{\text {type }}$ are strongly restricted.

In principle both bottom-up and top-down induction approaches can be used. Because of the ease of control, we decided to proceed top-down. Figure 1 shows our deterministic algorithm for determining argument types. It proceeds in two steps. 
Input: set $A$ of argument terms

Algorithm: argument_type $(A)$

(1) Determine heads:

$A_{0}:=\{T \in A \mid T$ atomic $\}$

$A_{f}:=\left\{T \in A \mid T=f\left(T_{1}, \ldots, T_{m}\right), m \geq 1\right\}$ for each $f$ occurring in $A$

Heads: for each $A_{f}$ a head $H_{f}=t_{A}\left(\lg g\left(A_{f}\right)\right)$

where $\lg g$ is the least general generalisation [Plo70]

(2) Top-down induction of bodies:

for each Head $H_{f}$

for each $V \in \operatorname{vars}\left(H_{f}\right)$

if $\exists p \in B_{t y p e}$ such that $p(V \sigma) \forall H_{f} \sigma \in A_{f}$

then add $p(V)$ to the body of $H_{f}$

else if $t_{x}(V \sigma) \forall H_{f} \sigma \in A_{f}$ where $t_{x}=t_{A}$ or $t_{x}$ calls $t_{A}$

then add $t_{x}(V)$ to the body of $H_{f}$

else $A^{\prime}=\left\{V \sigma \mid H_{f} \sigma \in A_{f}\right\}$

add $t_{A^{\prime}}(V)$ to the body of $H_{f}$

call argument_type $\left(A^{\prime}\right)$

Output: $t_{A}$

Fig. 1. Top-down algorithm for determining argument types

The first step determines clause heads for the target argument type $t_{A}$. The set $A$ is partitioned according to different functors, and for each partition its least general generalisation lgg [Plo70] is taken as head argument of $t_{A}$. The second step induces clause bodies defining $t_{A}$ by searching for type restrictions for the variables in each clause head $H_{f}$ of $t_{A}$. First, the background knowledge $B_{\text {type }}$ is checked. If a predicate $p$ is valid for all instantiations of $V$ in $A$, and $p$ is the most specific predicate in $B_{\text {type }}$ fulfilling this condition, $p(V)$ is added as body literal. Else, the same is tested for predicates $t_{x}$, where $t_{x}$ calls $t_{A}$ directly or via intermediate predicates $t_{z}$, or $x=A$. In the last case, $t_{A}$ is defined recursively.

If none of those applies, a literal $t_{A^{\prime}}(V)$ is added to the body of $H_{f}$ and the algorithm is called recursively on the set $A^{\prime}$ of all instantiations of $V$ in $A_{f}$.

Example 2. Let the set of argument terms occurring at an argument position in $E$ be $A=\{\operatorname{not}(\operatorname{and}([a])), \operatorname{not}(\operatorname{and}([x, y])), \operatorname{not}(\operatorname{and}([u, v, w]))\}=A_{\text {not }}$. The clause head determined for $A_{\text {not }}$ is

$$
\text { (1) } t_{A}\left(\operatorname{lgg}\left(A_{n o t}\right)\right)=t_{A}(\operatorname{not}(\operatorname{and}([X \mid Y]))) \text {. }
$$

A type restriction for both variables $X$ and $Y$ is to be found. As atom $(X)$ is true for the instantiations $a, x$ and $u$ of $X$ in $A_{n o t}$, it is added to the body of (1) yielding

$$
\left(1^{\prime}\right) t_{A}(\operatorname{not}(\operatorname{and}([X \mid Y]))) \leftarrow \operatorname{atom}(X) \text {. }
$$


For the instantiations of $Y$, neither atom, number, atomic nor a predicate $t_{x}$ calling $t_{A}$ is true. Therefore, a new literal $t_{A^{\prime}}(Y)$ is added to the body of $\left(1^{\prime}\right)$ resulting in

$$
\left(1^{\prime \prime}\right) t_{A}(\operatorname{not}(\operatorname{and}([X \mid Y]))) \leftarrow \operatorname{atom}(X), t_{A^{\prime}}(Y) .
$$

The algorithm is called recursively with the set $A^{\prime}=\{[,[y],[v, w]\}$ of $Y$ 's instantiations. $A^{\prime}$ contains the atomic term $[$ and the 2-place functor [-1].Therefore we get two sets $A_{0}^{\prime}=\left\{[\}\right.$ and $A_{[-1-]}^{\prime}=\{[y],[v, w]\}$ and, accordingly, two heads

$$
\begin{aligned}
& \text { (2) } t_{A^{\prime}}([) . \\
& \text { (3) } t_{A^{\prime}}([H \mid T]) \text {. }
\end{aligned}
$$

As (2) does not contain any variable, it is complete. For $H$ and $T$ further restrictions have to be determined. As above, $H$ is restricted by the condition atom $(H)$ yielding

$$
\left(3^{\prime}\right) t_{A^{\prime}}([H \mid T]) \leftarrow \operatorname{atom}(H) .
$$

For the instantiations $\left[\right.$ and $[w]$ of $T$ in $A_{[-1]}^{\prime}$ the predicate $t_{A^{\prime}}(T)$ defined by (2) and $\left(3^{\prime}\right)$ is valid such that we get a recursive definition of $t_{A^{\prime}}$. This completes the argument type definition for $A$ :

$$
\begin{aligned}
& \left(1^{\prime \prime}\right) t_{A}(\operatorname{not}(\operatorname{and}([X \mid Y]))) \leftarrow \operatorname{atom}(X), t_{A^{\prime}}(Y) . \\
& (2) t_{A^{\prime}}([) . \\
& \left(3^{\prime \prime}\right) t_{A^{\prime}}([H \mid T]) \leftarrow \operatorname{atom}(H), t_{A^{\prime}}(T) .
\end{aligned}
$$

Because of the small number of possible body literals the search space is small even for complex types, e.g. types containing more than one recursion like binary trees or types recurring over several steps.

A problem is how to induce type restrictions for predicates in the background knowledge. As usually no examples are supplied, our method is not applicable. In our experimental system, we chose the simplest solution and required those types to be given or learnt incrementally. However, there are alternative methods for computing the types of given programs, e.g. [Kluz87].

\section{Determining Clause Heads}

The algorithm in this section determines a set of clause heads partitioning the examples according to structural properties. In general, the least general generalisation [Plo70] of each subset of the example set is a candidate for being clause head of a disjunctive clause. As it is intractable to consider each subset and the corresponding clause head, we restrict our algorithm to subsets with the same argument structure and try to construct clause heads from them.

Given the set $E$ of positive examples for a predicate $p / n$, a mode declaration for $p / n$ [Sha83] and the type restriction

$$
\text { type_restriction }\left(p\left(X_{1}, \ldots, X_{n}\right),\left\{\text { type }_{1}\left(X_{1}\right), . ., \text { type }_{n}\left(X_{n}\right)\right\}\right)
$$


together with complete definitions of each $t_{y p e_{i}}$, our algorithm proceeds in three steps. First, it partitions the example set according to the type restriction by clustering examples with the same structure at an argument position. The least general generalisations of those partitions are refined further in the second step. The last step implements a subsumption-based reduction of the set of clause heads.

\subsection{Determining Clause Heads According to the Type Restriction}

The first step of the algorithm partitions the example set according to the different structures described in the type restriction. Different structures at a single argument position $i$ correspond to different proofs of the $i$ th argument being of type $_{i}$. To distinguish the different structures at position $i$, the proof structures for type $e_{i}$ have to be considered.

Let $C_{i}$ be the clauses defining type i $_{i}$ A set $S_{i} \subseteq C_{i}$ corresponds to a proof structure iff

- at least one clause $D \in S_{i}$ has type $e_{i}$ as head predicate

- for each $D \in S_{i}$, if $D$ calls type $_{j}$ then $S_{i}$ contains at least one clause with type $_{j}$ as head predicate.

That is, $S_{i}$ must conform the subgoal hierarchy of type $i$. As $S_{i} \subseteq C_{i}, 2^{\left|C_{i}\right|}$ is an upper bound for the number of possible proof structures. For each proof structure $S_{i}$ we define

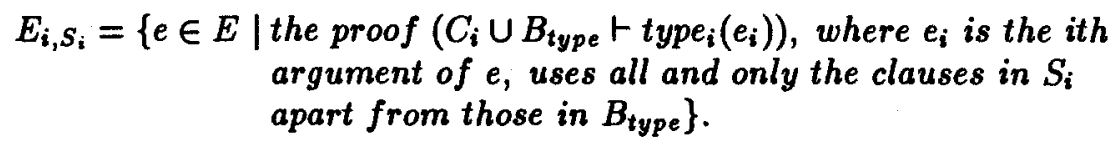

The sets $E_{i, S_{i}}$ contain examples with the same structure at the $i$-th argument position. For complex types, there may be a large number of those sets and accordingly a large number of clause heads. However, it might be the case that the heads for different sets $E_{i, S_{i}}$ coincide, as our example will show.

Example 3. Let the example set for merging two sorted lists of numbers in one be $E=\{$ merge $([,[]$,$) ,$

$$
\begin{aligned}
& \text { merge }([6],[],[6]), \\
& \text { merge }([,[7],[7]), \\
& \text { merge }([1],[2,4],[1,2,4]), \\
& \text { merge }([6,8],[5],[5,6,8])\} .
\end{aligned}
$$

The type restriction our algorithm determines is

$$
\text { type_restriction(merge } \left.(X, Y, Z),\left\{t_{1}(X), t_{1}(Y), t_{1}(Z)\right\}\right)
$$

with $t_{1}$ being defined as

$$
\begin{aligned}
C_{i}=\{1 & 1 t_{1}([) . \\
& \left.2 t_{1}([A \mid B]) \leftarrow \text { number }(A), t_{1}(B) . \quad\right\}
\end{aligned}
$$


For $i=1,2,3$ there are two possiblities for $S_{i}$, namely $\{1\}$ and $\{1,2\}$. The nonempty sets $E_{i, S_{i}}$ are

$E_{1,\{1\}}=\{\operatorname{merge}(\square, \square, \square), \operatorname{merge}(\square,[7],[7])\}$

$E_{2,\{1\}}=\{\operatorname{merge}([,[, 0), \operatorname{merge}([6],[,[6])\}$

$E_{3,\{1\}}=\{\operatorname{merge}(\bar{\square}, \mathbb{\square}, \bar{\square})\}$

$E_{1,\{1,2\}}=\{\operatorname{merge}([1],[2,4],[1,2,4]), \operatorname{merge}([6,8],[5],[5,6,8]), \operatorname{merge}([6], \square,[6])\}$

$E_{2,\{1,2\}}=\{\operatorname{merge}([1],[2,4],[1,2,4]), \operatorname{merge}([6,8],[5],[5,6,8]), \operatorname{merge}([],[7],[7])\}$

$E_{3,\{1,2\}}=\{\operatorname{merge}([1],[2,4],[1,2,4]), \operatorname{merge}([6,8],[5],[5,6,8]), \operatorname{merge}([6],[],[6])$, $\operatorname{merge}([,[7],[7])\}$

Base cases of structured argument terms correspond to proofs that use exactly one clause in $C_{i}$, e.g. the empty list [ is a base case of $t_{1}$ in our example as the proof of $t_{1}(\mathbb{Z})$ needs only clause 1 . For singleton sets $S_{i} E_{i, S_{i}}$ contains examples with base cases at argument position $i$. We define the set of all base examples as

$$
E_{\text {bases }}=\bigcup_{i \in\{1, . ., n\}} E_{i, S_{i}} \text { for } S_{i} \subseteq C_{i} \text { such that }\left|S_{i}\right|=1
$$

With those definitions the first set of clause heads is defined as

$$
H=H_{\text {bases }} \cup H_{s t r u c t u r e d}
$$

where

$$
H_{\text {bases }}=\bigcup_{i \in\{1, . ., n\}}\left\{\operatorname{lgg}\left(E_{i, S_{i}}\right)\left|S_{i} \subseteq C_{i} \wedge\right| S_{i} \mid=1 \wedge E_{i, S_{i}} \neq \phi\right\}
$$

and

$$
H_{\text {structured }}=\bigcup_{i \in\{1, . ., n\}}\left\{\operatorname{lgg}\left(E_{i, S_{i}}-E_{b a s e s}\right)\left|S_{i} \subseteq C_{i} \wedge\right| S_{i} \mid \geq 2 \wedge E_{i, S_{i}} \neq \phi\right\}
$$

Example 4. Continuing the previous example, we get the following clause heads:

$$
\begin{aligned}
& H_{\text {bases }} \quad i=1 \mid \operatorname{lgg}(\{\operatorname{merge}(\square, \square, \square), \operatorname{merge}(\square,[7],[7])\})=\operatorname{merge}(\square, L, L) \\
& S_{i}=\{1\} \quad i=2 \operatorname{lgg}(\{\operatorname{merge}(\square, \square, \square), \operatorname{merge}([6], \square,[6])\})=\operatorname{merge}(L, \square, L) \\
& i=3 \mid \operatorname{lgg}(\{\operatorname{merge}(\square, \square, \square)\})=\operatorname{merge}(\square, \square, \square) \\
& \begin{array}{l}
H_{\text {structured }} \\
S_{i}=\{1,2\}|i=1| \begin{array}{l}
\operatorname{lgg}(\{\operatorname{merge}([1],[2,4],[1,2,4]), \operatorname{merge}([6,8],[5],[5,6,8])\}) \\
=\operatorname{merge}([A \mid B],[C \mid D],[E, F \mid G]) \\
\operatorname{lgg}(\{\operatorname{merge}([1],[2,4],[1,2,4]), \operatorname{merge}([6,8],[5],[5,6,8])\}) \\
=\operatorname{merge}([A \mid B],[C \mid D],[E, F \mid G])
\end{array} \\
\left.\begin{array}{l}
= \\
\operatorname{lgg}(\{\operatorname{merge}([1],[2,4],[1,2,4]), \operatorname{merge}([6,8],[5],[5,6,8])\}) \\
=\operatorname{merge}([A \mid B],[C \mid D],[E, F \mid G])
\end{array}\right)
\end{array}
\end{aligned}
$$




\subsection{Refining the Set of Clause Heads}

Though the set of clause heads determined in the previous step partitions the examples according to their overall structure, further refinements are possible. A useful refinement is the search for equal structures at different argument positions. This is done by unifying terms within a clause head. In order to restrict the possible unifications we use Wirth and O'Rorke's heuristic of critical variables [WO91].

Definition 1. Let $H$ be a clause head with variables $V_{H}$. The critical variables $V c_{H}$ of $H$ are all input variables that occur in $H$ only once and all output variables that do not occur as input variables

Critical terms are all terms in $H$ that contain only critical variables and no atoms except for the base cases of structured types.

E.g., given merge $(+,+,-)$ as mode declaration, the critical input terms of merge $([A \mid B],[C \mid D],[A \mid E])$ are $\{B, C, D,[C \mid D]\}$ and the only critical output term is $E$.

Figure 2 shows the algorithm for refining the set of clause heads. Given a clause head $h$ and a pair of critical terms of the same type within $h$, the algorithm tests whether the head $h^{\prime}$ resulting from unifying the terms covers some examples. If this is the case, the least general generalisation of that examples is taken as a further head, and the algorithm is recursively applied to it. The algorithm stops if no further heads result from the unifications.

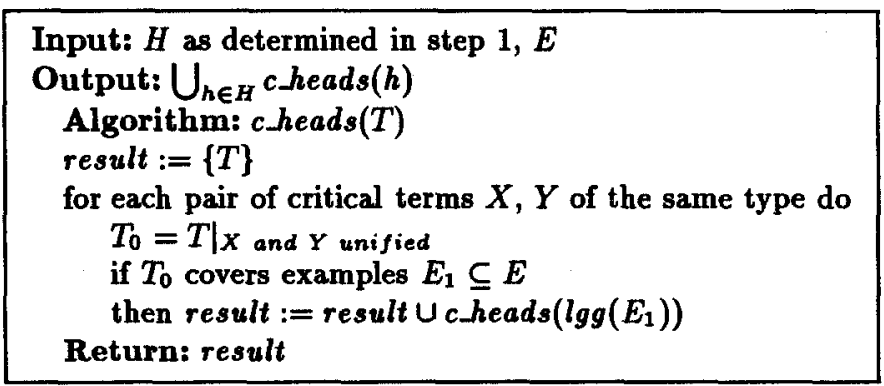

Fig. 2. Algorithm for refining clause heads

E.g. unifying the critical terms $A$ and $E$ in merge $([A \mid B],[C \mid D],[E, F \mid G])$ yields a more specific head merge $([A \mid B],[C \mid D],[A, F \mid G])$ covering examples like merge $([1,2],[4],[1,2,4])$. Extending this branch by unifying $C$ and $F$, we get merge $([A \mid B],[C \mid D],[A, C \mid G])$, and at last merge $([A],[C \mid D],[A, C \mid D])$ by unifying $D$ and $G$. As this head contains no more critical terms, the algorithm stops on this branch.

Figure 3 shows the clause heads our algorithm determines starting with the head merge $([A \mid B],[C \mid D],[E, F \mid G])$ according to the example set we gave. If 
different examples are given, the algorithm may result in further heads indicated by the dashed lines. The example shows that the resulting set of clause heads is generally redundant and can be minimized.

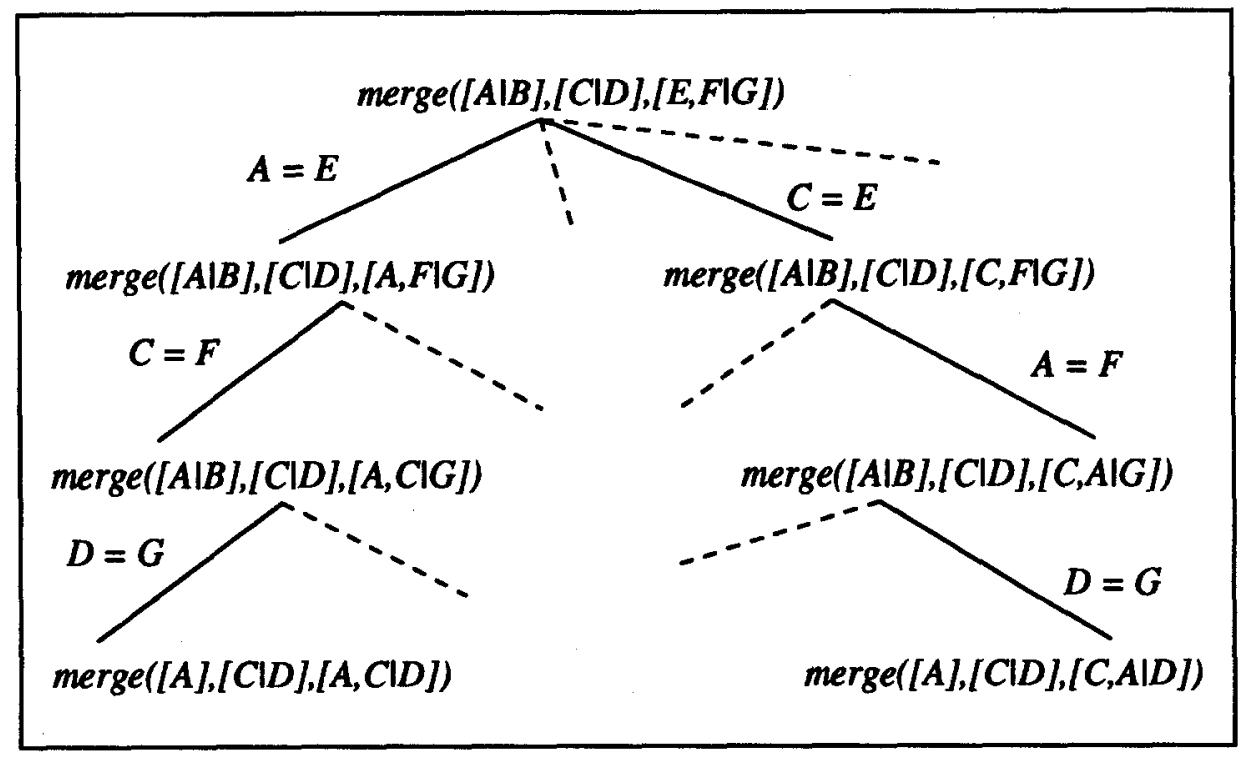

Fig. 3. Non-base clause heads for merge

\subsection{Minimizing the Set of Clause Heads}

The set of clause heads determined in the previous step is redundant in two ways. It contains both heads that are too general and heads that are too specific. The aim of this step is to remove redundant heads. For that aim, we developed a simple heuristical subsumption-based method. Let $G$ be the graph spanned by $\theta$-subsumption on the set $H$ of clause heads, i.e.

$$
G=(H, A) \text { where } A=\left\{\left(H_{i}, H_{j}\right) \in H \times H \mid H_{i} \neq H_{j} \wedge \exists \sigma H_{j} \sigma=H_{i}\right\} .
$$

The first step of our method removes all redundant heads starting with the most general ones in $G$. The second step eliminates the redundant heads starting with the most specific ones in the remaining graph. Figure 4 shows the algorithm more formally.

Example 5. The clause heads determined for merge yield the graph in figure 5 according to $\theta$-subsumption.

In step 1 of our algorithm head (4) is removed as a most general redundant head. In the reduced graph there are no more most general redundant heads, 


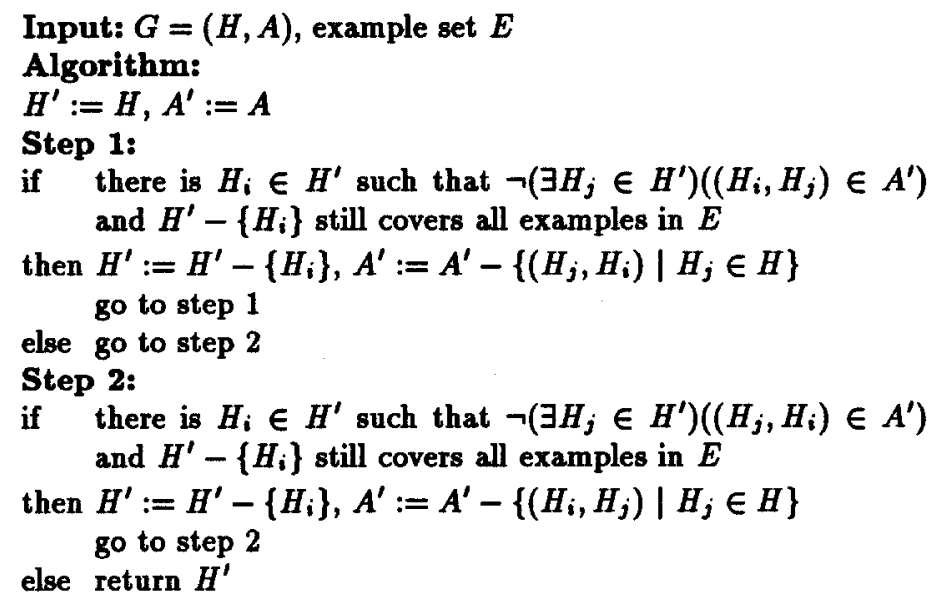

Fig. 4. Algorithm for optimizing the set of clause heads

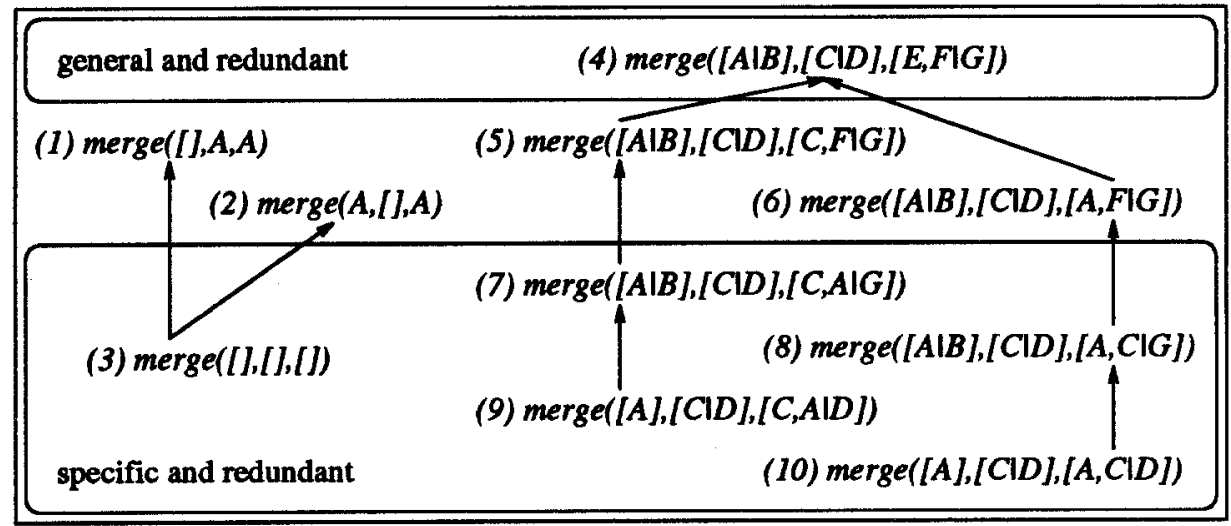

Fig. 5. Optimizing the clause heads for merge

and the algorithm proceeds to step 2. This step removes successively the most specific redundant heads (3), (9), (10), (7) and (8). The remaining set $H^{\prime}$ consists of the heads (1), (2),(5) and (6).

Our method worked surprisingly well on our examples. However, there are simple counterexamples where it removes heads necessary for the subsequent learning process. E.g. the first head in the set $\{\min (X,[X]), \min (X,[Y \mid R])\}$ is removed though it is a necessary base case. The method should be adapted to recognize and retain base cases of that kind.

The most similar approach to our partitioning algorithm is the method of Arimura, Shinohara and Otsuki [ASO91]. Given a set of positive examples, it produces a non-redundant set of clause heads covering the example set. In con- 
trast to our algorithm, it works in polynomial time. However, it is restricted on producing at most two different clause heads for the example set. The extension of the method to arbitrary many heads is not straightforward and may lead to exponential running times.

In the following section we show how the optimized set of clause heads is employed during top-down induction in our system INDICo.

\section{Overview of INDICO}

The setting for our sytem INDICo is as follows: Given a set $E$ of positive examples for a functional predicate $p / n$, a mode declaration for $p / n$ [Sha83] and a logic program $B$ as background knowledge, the learning task is to find a set of clauses $P$ such that $B \cup P$ computes the correct output for each of the examples in $E$.

INDICO proceeds in three steps. First, it determines the argument types of the target predicate by means of our algorithm described in section 2 . Then, it computes the minimized set of clause heads using the second algorithm we discussed in this paper. This set of clause heads is taken as possibly overgeneral Prolog-program for the target predicate. In the third step, InDICo locates overgeneral clauses within the preliminary program and specialises them by adding literals, including newly invented ones, to their bodies.

Note that any other top-down inductive learner could use the clause heads INDICO determined. For example, FoIl could be supplied with the head showing the largest information gain [Qui90] on the example set as starting point of its search.

The method INDICo employs for inducing clause bodies is discussed in detail in [STW91]. As we restricted INDICo to positive examples as input only, the crucial problem during the third step is how overgeneralisation can be detected in the absence of negative examples. In [STW91] we describe a method for constructing negative examples without the completeness restriction on positive examples that must hold for the application of the closed world assumption. This method is suitable only for a restricted class of functional predicates. However, the restriction on functional predicates is not necessary for applying the algorithms described in section 2 and 3.

The negative examples are used for localizing overgeneral clauses and specialising them by adding a literal to their body. The body literals can be background predicates, the target predicate itself and newly invented predicates. The search space is strongly constrained by type restrictions, restrictions on data flow, correctness conditions and a heuristic measure combined with beam search. In doing so, ideas from MIS [Sha83], CHAM [Kij92], and SiERES [WO91] have been used.

\section{Experimental Results}

INDICO has been tested on several examples including logic programs operating on lists like append, merge, split, reverse, partition and sort. For each of those, 
it performed well in detecting the main structural distinctions in the example set.

A more complex class of example programs is a set of logic programs operating on ordered binary trees. Binary trees are represented as lists [left_subtree, root, right_subtree], where root is a number and left_- and right_subtree are binary trees. The empty tree is [].

INDICO has been able to induce definitions for inserting an element in an ordered binary tree in the appropriate position and, as a more difficult example, for retracting an element from the tree such that the ordering is maintained. This may require rotations in the tree, as illustrated in figure 6 . For retracting

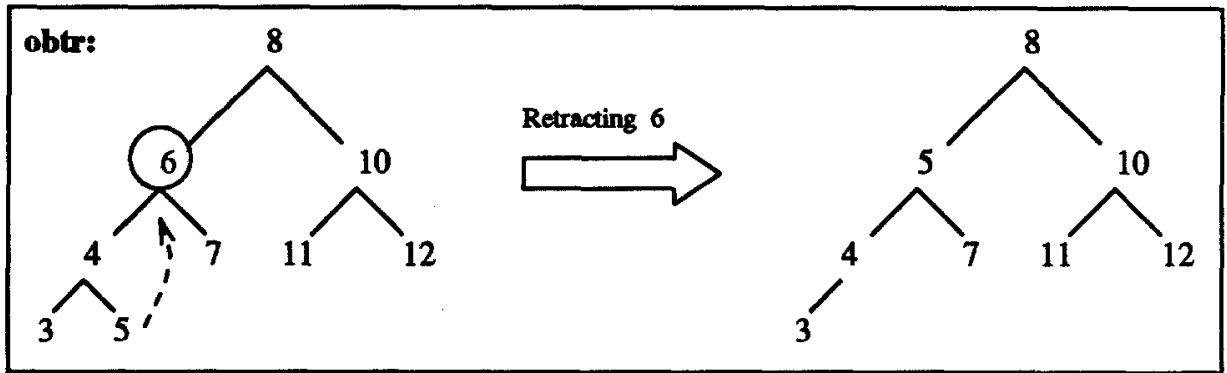

Fig. 6. Retracting an element from an ordered binary tree

an element from a tree INDICo discovered the main disjunctive cases in the example set as far as structure is concerned: the two cases that the left or right subtree of the element to be deleted is empty, the case that both subtrees of the element are not empty and a suitable element for replacing the retracted element has to be found in the tree, and the two recursive cases where the element to be deleted is not the root of the actual tree, but has to be deleted in the left or right subtree.

Given this partitioning of the 21 examples we supplied for obtr, INDICo induces the following definition:

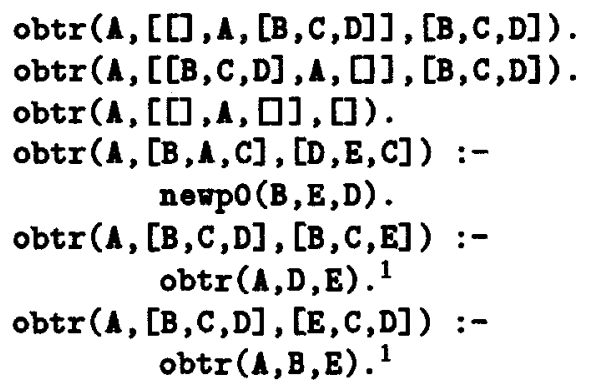

newpo $([[A, B, C], D,[]], D,[A, B, C])$. nexpo $([], \mathbf{A},[]], \mathbf{A}, \square)$.

newpo $([A, B, C], D,[A, B, E]):-$ newpo $(C, D, E)$.

1 The definition of the recursive cases does not check whether $A$ is contained in the left or in the right subtree. As obtr fails if the element to be deleted does not occur in the tree, this is merely a difference in efficiency. 
For the case that both subtrees of the element to be deleted are not empty, INDICo invented a new predicate which locates a suitable element for replacing the element to be deleted and performs the rotation.

Our examples show that the algorithm is reasonable efficient and powerful in inducing Horn clause definitions in structured domains.

\begin{tabular}{|c|c|c|c|c|c|}
\hline & & $\begin{array}{l}\text { \# exam } \\
\text { pos. }\end{array}$ & $\begin{array}{l}\text { ples } \\
\text { neg. }\end{array}$ & $\begin{array}{c}\| \text { tested } \\
\text { hypotheses }\end{array}$ & $\begin{array}{l}\text { background } \\
\text { knowledge }\end{array}$ \\
\hline \multirow[t]{4}{*}{ append } & CHAM & 39 & 365 & 142 & none \\
\hline & Foll & 261 & 8991 & 1018 & components, null, list \\
\hline & MIS & $1+28^{2}$ & - & 144 & none \\
\hline & INDICo & 13 & $(1)^{3}$ & $6 / 5^{4}$ & none \\
\hline \multirow[t]{4}{*}{ reverse } & CHAM & 16 & 256 & 167 & addlast \\
\hline & FoIL & 65 & 4225 & 299 & $\begin{array}{c}\text { components, null, list } \\
\text { append }\end{array}$ \\
\hline & MIS & $1+5$ & $=$ & 66 & addlast induced \\
\hline & INDICO & $11+8$ & (1) & $3 / 6$ & addlast invented \\
\hline \multirow[t]{4}{*}{ isort } & CHAM & 23 & 17 & $\overline{325}$ & part, append, component \\
\hline & FOIL & 16 & 256 & 201 & insert, null, component \\
\hline & MIS & $1+6$ & $=$ & 56 & $<$, insert induced \\
\hline & $\overline{\text { INDICO }}$ & $11+8$ & (1) & $5 / 6$ & $<$, insert invented \\
\hline
\end{tabular}

Table 1. Test results

At last, we want to compare our system INDICo with MIS [Sha83], FoIL [Qui90] and CHAM [Kij92] running on similar tasks in order to measure the improved efficiency resulting from our problem reduction approach. The selection of these tasks was a difficult problem since all systems differ quite strongly in the predicates they are able to learn. Therefore we restricted our tests on three list-manipulating predicates, namely append, reverse and sort. Those tests are of course far from being representative for the different behaviour of the systems, but they give some idea of it.

Another problem with the tests was which evaluation criteria to chose. As running times are meaningless - especially since FoIL is C-encoded and the rest in QUINTUSProLOG - we took the number of examples needed, the background knowledge $B$ supplied and the number of hypotheses generated and tested.

Each system was supplied the examples provided by its authors. We assumed those to be the best-suited for the learning task. E.g. due to the information gain

$2 x+y$ means that the system was given $x$ examples and asked $y$ membership- or existential queries

${ }^{3}$ Parenthesized numbers of negative examples refer to the examples INDICO constructs from positive examples as described in [STW91].

4 Here, $x / y$ means that INDICO generated $x$ clause heads and, for the selected set of clause heads, $y$ different body hypotheses. 
heuristic, FolL [Qui90] needs more examples than MIS [Sha83]. Concerning the number of examples, INDICO ranges between MIS and CHAM. It does not need as carefully chosen examples as MIS. However, they must not be as arbitrary as for Foll. For each disjunct, at least two examples must be given, and the examples should be simple. That is, the learning behaviour of INDICo deteriorates if e.g. append is exemplified only for lists with a length above 1000 .

For MIs, Foll and Cham the number of hypotheses is exactly the number of clauses that have been searched. For INDico, both the number of clause heads the system generated and the number of body hypotheses are listed. However, we do not list the amount of search INDICo performs for determining argument types, as Mis, FoIL and CHAM employ user-supplied type declarations.

The results of our experiments are illustrated in table 1 . Note that there is a difference between MIS and INDICo in inferring the necessary background knowledge as e.g. for isort or reverse. MIS asks the oracle which predicates it may use for constructing a clause body and, if not yet defined, induces their definition. INDICO proceeds differently: it does not know which predicates to use for constructing a clause body. It realizes the point at which a new predicate is needed and extends its vocabulary at that point. Unlike MIs, the user does not have to supply the system with examples for the new predicate.

The results in table 1 show that the problem reduction method is successfully applicable to the problem of inductive inference. Due to the constraints resulting from the clause heads, INDICO searches the fewest hypotheses and is additionally capable of inventing new predicates if the existing background knowledge is not sufficient. Furthermore, the amount of work for determining the clause heads is small and so there is a real increase in overall efficiency. If the other systems integrated the structural constraints from the partitioning of the example set, they would have much less hypotheses to generate and test.

\section{Conclusions}

The current implementation of our algorithm shows that reducing the problem of top-down inductive inference to the simpler subproblems of determining clause heads and clause bodies improves the efficiency of top-down inductive learners considerably. The constraints emerging from the clause heads and the type restrictions can be exploited during the induction of the clause bodies and help systems in finding definitions more efficiently or, for complex definitions, even in finding them at all.

In our system INDICO we use the algorithm for determining clause heads as a real alternative to the covering approach, as the optimized set of clause heads is taken as complete but possibly overgeneral program for the target predicate. It would be interesting to integrate it with a greedy algorithm for inducing clauses, e.g. FolL, and investigate the resulting improvement in efficiency and power. 


\section{References}

[ASO91] Arimura, H., Shinohara, T., Otsuki, S. (1991): Polynomial Time Inference of Unions of Tree Pattern Languages in Proc. of the 2nd Workshop on Algorithmic Learning Theory, Tokyo

[Kij92] Kijsirikul, B., Numao, M., Shimura, M. (1992): Efficient Learning of Logic Programs with Non-determinate, Non-dicriminating Literals in S. Muggleton (ed): Inductive Logic Programming, Academic Press

[Kluz87] Kluzniak, F. (1987): Type Synthesis for Ground Prolog, in J. L. Lasses (ed): Logic Programming, Proc. of the 4th International Conference, MIT-Press

[Plo70] Plotkin, G. D. (1970): A Note on Inductive Generalisation in: B. Meltzer, D. Mitchie (eds): Machine Intelligence 5, Edinburgh University Press.

[Qui90] Quinlan, J. R. (1990): Learning Logical Definitions from Relations, Machine Learning 5, $239-266$.

[Sha83] Shapiro, E. Y. (1983): Algorithmic Program Debugging, MIT Press, Cambridge, Mass.

[Sta91] Stahl, I. (1991): Induktion von disjunktiven Konzepten, Diplomarbeit Nr. 807, Universität Stuttgart, Fakultät Informatik.

[STW91] Stahl, I., Tausend, B., Wirth, R. (1991): General-to-specific Learning of Horn Clauses from Positive Examples, in Proc. of the CompEuro92, The Hague, NL

[WO91] Wirth, R., O'Rorke, P. (1991): Constraints on Predicate Invention, Proc. of the 8th International Workshop on ML, Chicago, Morgan Kaufmann.

[YS91] Yardeni, E., Shapiro, E. (1991): A Type System for Logic Programs, Journal of Logic Programming 10. 